\title{
Video Article \\ A Multilayer Microfluidic Platform for the Conduction of Prolonged Cell-Free Gene Expression
}

\author{
Ardjan J. van der Linden ${ }^{1}$, Maaruthy Yelleswarapu ${ }^{2}$, Pascal A. Pieters ${ }^{1}$, Zoe Swank ${ }^{3}$, Wilhelm T. S. Huck ${ }^{2}$, Sebastian J. Maerkl ${ }^{3}$, Tom F. A. de \\ Greef $^{1,2}$ \\ ${ }^{1}$ Institute for Complex Molecular Systems, Department of Biomedical Engineering, Computational Biology Group, Eindhoven University of Technology \\ ${ }^{2}$ Institute for Molecules and Materials, Radboud University \\ ${ }^{3}$ Institute of Bioengineering, School of Engineering École Polytechnique Fédérale de Lausanne (EPFL)
}

Correspondence to: Sebastian J. Maerkl at sebastian.maerkl@epfl.ch, Tom F. A. de Greef at t.f.a.d.greef@tue.nl

URL: https://www.jove.com/video/59655

DOI: doi:10.3791/59655

Keywords: Bioengineering, Issue 152, Microfluidics, in vitro transcription and translation, synthetic biology, prolonged reactions, micro-reactors, protein expression

Date Published: 10/6/2019

Citation: van der Linden, A.J., Yelleswarapu, M., Pieters, P.A., Swank, Z., Huck, W.T., Maerkl, S.J., de Greef, T.F. A Multilayer Microfluidic Platform for the Conduction of Prolonged Cell-Free Gene Expression. J. Vis. Exp. (152), e59655, doi:10.3791/59655 (2019).

\section{Abstract}

The limitations of cell-based synthetic biology are becoming increasingly apparent as researchers aim to develop larger and more complex synthetic genetic regulatory circuits. The analysis of synthetic genetic regulatory networks in vivo is time consuming and suffers from a lack of environmental control, with exogenous synthetic components interacting with host processes resulting in undesired behavior. To overcome these issues, cell-free characterization of novel circuitry is becoming more prevalent. In vitro transcription and translation (IVTT) mixtures allow the regulation of the experimental environment and can be optimized for each unique system. The protocols presented here detail the fabrication of a multilayer microfluidic device that can be utilized to sustain IVTT reactions for prolonged durations. In contrast to batch reactions, where resources are depleted over time and (by-) products accumulate, the use of microfluidic devices allows the replenishment of resources as well as the removal of reaction products. In this manner, the cellular environment is emulated by maintaining an out-of-equilibrium environment in which the dynamic behavior of gene circuits can be investigated over extended periods of time. To fully exploit the multilayer microfluidic device, hardware and software have been integrated to automate the IVTT reactions. By combining IVTT reactions with the microfluidic platform presented here, it becomes possible to comprehensively analyze complex network behaviors, furthering our understanding of the mechanisms that regulate cellular processes.

\section{Video Link}

The video component of this article can be found at https://www.jove.com/video/59655/

\section{Introduction}

Cells are able to sense and respond to their environment using complex dynamic regulatory networks ${ }^{1,2}$. The field of synthetic biology utilizes our knowledge of the naturally occurring components comprising these networks to engineer biological systems that can expand the functionality of cells ${ }^{3,4}$. Conversely, it is also possible to further our understanding of the natural networks governing life by designing simplified, synthetic analogues of existing circuits or by forward-engineering biological systems which exhibit naturally occurring behaviors. The de novo engineering of such biological systems is performed in a bottom-up fashion where novel genetic circuits or signalling pathways are engineered in a rational manner, using well-defined parts ${ }^{5,6}$. Combining the rational design of networks with the design of biologically relevant systems enables the indepth characterization and study of biological regulatory systems with various levels of abstraction ${ }^{7}$.

The pioneering works of Elowitz and Leibler ${ }^{8}$ and Gardner et al. ${ }^{9}$ were the first to demonstrate the successful introduction of synthetic genetic networks into cellular hosts. In the following decade, numerous researchers have continued to build on these initial successes despite the emergence of several limitations regarding the introduction of synthetic circuits into cells $\mathbf{s}^{7,10,11,12}$. Ideally, the introduction of synthetic circuits into cellular hosts should occur in a modular fashion. Unfortunately, the complexity of the cellular environment makes this particularly challenging, with the function of many parts and networks being highly context dependent ${ }^{12,13,14}$. As a result, networks often experience undesired interactions with native host componentry which can affect the function of the synthetic circuit. Similarly, components of the exogenous network can inhibit host processes, compete for shared resources within the host, and influence growth kinetics ${ }^{15,16,17}$. Consequently, in order to rationally design and predict the behavior of synthetic networks in an in vivo environment, a comprehensive model of all host and circuit-specific dynamics is required ${ }^{18}$

A viable alternative to the use of cellular hosts for the characterization of synthetic networks is the application of in vitro transcription and translation (IVTT) technologies. Acting as a testbed for synthetic networks, reactions are performed in solutions comprising all the components required to enable gene expression ${ }^{19,20,21}$. In this manner, a biologically relevant, albeit artificial, environment is created within which synthetic networks can be tested $22,23,24,25,26,27,28$. A major advantage of using IVTT solutions is the ability to perform reactions under user-specified 
conditions, with researchers able to tune the precise composition of each reaction ${ }^{2}$. Furthermore, the cell-free approach enables high-throughput testing of synthetic networks, since it removes the need to perform time-consuming cellular cloning steps. As a result, the duration of successive design - build - test cycles is significantly reduced ${ }^{29,30,31,32}$. The design cycle can be further accelerated by utilizing cell-free cloning techniques such as the Gibson assembly to rapidly engineer novel networks, and by constructing networks from linear DNA templates which - unlike the plasmids required for in vivo testing - can be amplified via polymerase chain reactions (PCR) ${ }^{33,34}$

Batch reactions are the simplest method by which IVTT reactions can be performed, requiring a single reaction vessel wherein all of the reaction components are combined ${ }^{35}$. Such reactions are sufficient for protein expression and basic circuit testing yet prove insufficient when attempting to study the long-term dynamic behavior of a network. Over the course of a batch reaction, reagents are either depleted or undergo degradation resulting in a continuous decrease of the transcription and translation rates. Furthermore, as reactions progress by-products accumulate that can interfere with - or completely inhibit - the correct functioning of the network. Ultimately, the use of batch reactors limits the dynamic behavior which can be observed, with negative regulation being particularly challenging to implement ${ }^{5,36}$.

The versatility of IVTT systems enables multiple alternative methods by which prolonged IVTT reactions can be performed, ranging from continuous flow to droplet based methods as well as simpler dialysis approaches ${ }^{2,30,37,38,39,40}$. The application of microfluidic devices offers users increased control over their reactions whilst increasing the throughput and minimizing costs ${ }^{35,41,42}$, with each specific approach having its own advantages. The use of continuous flow can be easily optimized for increasing expression yields however, the inability to effectively remove specific reaction products makes the study of dynamic behavior non-trivial ${ }^{39}$. Whilst employing droplet based microfluidic systems allows high-throughput screening of novel networks, the difficulty of supplying fresh reagents to the reaction results in the droplets resembling small volume batch reactions ${ }^{43}$. Dialysis based reactors allow the introduction of fresh reagents as well as the removal of some reaction products however, RNA molecules and larger proteins accumulate within the reactor, being too large to diffuse through the membrane pores. Furthermore, large volumes of reagents are required to sustain these reactions for prolonged periods ${ }^{30,44}$. In 2013 , Maerkl et al. presented a multi-layered microfluidic device designed specifically for conducting prolonged IVTT reactions ${ }^{36,45}$. The use of multi-layered microfluidic devices permits direct control over fluid flow, allowing for the redirection of flow as well as the isolation of fluid in specific regions of the device ${ }^{46,47}$. These isolated regions can function as independent nanoliter-scale reaction chambers wherein IVTT reactions can be performed. Over the course of a single IVTT reaction, periodic injections of fresh reagents into the reactor are used to replenish IVTT components and DNA templates. Simultaneously, an equal volume of the old reaction solution is displaced, removing reaction products. In this manner, an out-of-equilibrium environment is maintained where the basal transcription and translation rates remain in steady-state, prolonging the lifetime of IVTT reactions and allowing rich dynamic behaviors to occur. By applying this approach, researchers are able to investigate the kinetic rates of the individual processes occurring within a specific circuit, aiding in the forward-engineering of novel genetic networks. For instance, Niederholtmeyer et al. implemented this approach to characterize various elements of a genetic ring oscillator, determining the kinetic rates thereof ${ }^{36}$. In further studies, Yelleswarapu et al. showed that the kinetic rates of sigma factor $28\left(\sigma_{28}\right)$ determined under batch conditions were insufficient to describe the behavior of a $\sigma_{28}{ }^{-}$ based oscillator, and that the addition of flow-based data improved model predictions of the network behavior ${ }^{22}$.

The goal of this manuscript is to present a complete protocol for the fabrication of multilayer microfluidic devices capable of performing longterm IVTT reactions. In addition, this manuscript will describe all of the hardware and software required to perform prolonged IVTT reactions. The actuation of the microfluidic device - necessary to control the flow of fluids therein - is achieved using a series of pneumatic valves which connect directly to the microfluidic devices via lengths of tubing. In turn, the pneumatic valves are controlled via a custom-built virtual control interface. Fluid flow within the microfluidic devices is achieved using continuous pressure which is provided by a commercially available pressure regulation system. IVTT reactions are typically performed between $29^{\circ} \mathrm{C}$ and $37^{\circ} \mathrm{C}$ and a microscope incubator is used to regulate the temperature during reactions. However, the functionality of the IVTT mixture gradually degrades when stored above $4{ }^{\circ} \mathrm{C}$. As such, this manuscript will expand on the off-chip cooling system used to cool the IVTT mixture prior to injection into the microfluidic device. In conclusion, this manuscript provides a comprehensive overview of the procedures required to successfully perform prolonged IVTT reactions using a microfluidic flow reactor such that other researchers will be able to replicate this technology with relative ease.

Protocol

\section{Wafer preparation}

NOTE: Our protocols are specific for the 40 XT positive photoresist and SU8 3050 negative photoresist used during this research. Alternative photoresists can be used, however the specific spin speeds, baking temperatures, and baking times will vary. The microfluidic device design provided by Niederholtmeyer et al. ${ }^{36}$ is linked in the Table of Materials.

1. Place two clean silicon wafers ( $100 \mathrm{~mm}$ diameter, $<1-0-0>$ oriented, $525 \mu \mathrm{m}$ thickness) in a preheated oven set to $250{ }^{\circ} \mathrm{C}$ and leave the wafers to dehydrate overnight $(\sim 16 \mathrm{~h})$.

NOTE: It is also possible to prime the wafers using HMDS vapor deposition; however, this is not necessary if the wafers are sufficiently dehydrated.

2. Remove one silicon wafer from the oven and let it cool to room temperature before proceeding with the spin coating. Apply $3-4 \mathrm{~mL}$ of the 40 $\mathrm{XT}$ photoresist to the center of the wafer.

3. To obtain a feature height of $25 \mu \mathrm{m}$ apply the following spin protocol: spin for $20 \mathrm{~s}$ at $500 \mathrm{rpm}$ (110 rpm/s), increase the spin speed to 3100 $\mathrm{rpm}(300 \mathrm{rpm} / \mathrm{s})$ and hold here for $30 \mathrm{~s}$, and decelerate the wafer to $0 \mathrm{rpm}$ with a deceleration of $200 \mathrm{rpm} / \mathrm{s}$. Using a microfiber tissue carefully remove any edge beading which may have occurred during the spin coating.

4. Soft bake using two separate hot plates set to $70^{\circ} \mathrm{C}$ and $120^{\circ} \mathrm{C}$ in the following manner: allow the wafer to sit at $70{ }^{\circ} \mathrm{C}$ for $30 \mathrm{~s}$. Then transfer the wafer to the $120^{\circ} \mathrm{C}$ hotplate and allow it to rest here for $3.5 \mathrm{~min}$ before returning the wafer to the $70^{\circ} \mathrm{C}$ hotplate for another $30 \mathrm{~s}$. Remove the wafer from the hotplate and - avoiding sudden temperature shocks - allow it to cool to room temperature.

5. Place the flow layer photomask (emulsion side down) onto the photoresist film and expose the wafer using a UV lamp until a total exposure of $200 \mathrm{~mJ} / \mathrm{cm}^{2}$ is achieved. 
6. Post-exposure bake using two hot plates $\left(70^{\circ} \mathrm{C}\right.$ and $\left.105^{\circ} \mathrm{C}\right)$ in the following manner: allow the wafer to sit at $70{ }^{\circ} \mathrm{C}$ for $20 \mathrm{~s}$ before transferring the wafer to the $105^{\circ} \mathrm{C}$ hotplate and leaving it here for $40 \mathrm{~s}$. Finally return the wafer to the $70{ }^{\circ} \mathrm{C}$ hotplate for a further $20 \mathrm{~s}$ to complete the post-exposure bake.

7. Allow the wafer to cool to room temperature on a stack of microfiber tissues. Develop the wafer by transferring it to a Petri dish filled with 726 MIF photoresist developer to start the development process. Development is accelerated when performed on a benchtop shaker and the entire wafer is submerged in the developer.

8. Rinse the wafer with demineralized water and use a stereo microscope to check the wafer surface for any photoresist residue. If photoresist residue can be seen, then return the wafer to the developer.

9. Reflow the positive photoresist by placing the wafer on a hot plate set at $110^{\circ} \mathrm{C}$ for $25 \mathrm{~min}$. This process will result in rounded features as well as annealing any cracks that may have appeared during the fabrication process. Proceed to silanize the wafer as described in step 1.17 .

10. Remove the second silicon wafer from the oven, allowing it to cool to room temperature before proceeding with the spin coating. Apply $5 \mathrm{~mL}$ of SU8 3050 photoresist to the center of the wafer.

11. To obtain a feature height of $30 \mu \mathrm{m}$ apply the following spin protocol: spin for $20 \mathrm{~s}$ at $500 \mathrm{rpm}(110 \mathrm{rpm} / \mathrm{s})$, increase the spin speed to 4,000 $\mathrm{rpm}(330 \mathrm{rpm} / \mathrm{s})$ and hold here for $42 \mathrm{~s}$, and decelerate the wafer to $0 \mathrm{rpm}$ with a deceleration of $200 \mathrm{rpm} / \mathrm{s}$. Using a microfiber tissue carefully remove any edge beading which may have occurred during the spin coating.

12. Soft bake using two separate hot plates $\left(65^{\circ} \mathrm{C}\right.$ and $\left.95^{\circ} \mathrm{C}\right)$ in the following manner: allow the wafer to sit at $65{ }^{\circ} \mathrm{C}$ for $30 \mathrm{~s}$. Then transfer the wafer to the $95^{\circ} \mathrm{C}$ hotplate and allow it to rest here for 14 min before returning the wafer to the $65^{\circ} \mathrm{C}$ hotplate for another $30 \mathrm{~s}$. Remove the wafer from the hotplate and allow it to cool to room temperature.

13. Measure the intensity of the UV lamp before exposure and use this to determine the exposure duration required to achieve a total exposure dosage of $260 \mathrm{~mJ} / \mathrm{cm}^{2}$. Place the photomask (emulsion side down) onto the photoresist film and place the wafer underneath the UV light source. Expose the wafer using a UV lamp until a total exposure of $260 \mathrm{~mJ} / \mathrm{cm}^{2}$ is achieved.

14. Post-exposure bake using two hot plates $\left(65^{\circ} \mathrm{C}\right.$ and $\left.95^{\circ} \mathrm{C}\right)$ in the following manner: allow the wafer to sit at $65^{\circ} \mathrm{C}$ for $60 \mathrm{~s}$ before transferring the wafer to the $95{ }^{\circ} \mathrm{C}$ hotplate and leaving it here for $4.5 \mathrm{~min}$. Return the wafer to the $65^{\circ} \mathrm{C}$ hotplate for a further $30 \mathrm{~s}$ to complete the postexposure bake.

15. Allow the wafer to cool to room temperature on a stack of microfiber tissues. Develop the wafer by transferring it to a Petri dish filled with $\mathrm{mrDev}-600$ photodeveloper to start the development process. Development is accelerated when performed on a benchtop shaker and the entire wafer is submerged in the developer.

16. Rinse the wafer with isopropanol and use a stereo microscope to check the wafer surface for any photoresist residue. If photoresist residue can be seen, then return the wafer to the developer. Once fully developed, hard bake the photoresist by placing the wafer on a hot plate set at $150{ }^{\circ} \mathrm{C}$ for $1 \mathrm{~h}$.

17. Silanize both wafers to prevent the adhesion of PDMS during the soft-lithography processes. To perform the silanization, pipet 2-3 droplets (per wafer) of the silane into a small glass vial. Place this vial, along with the wafers into a desiccator and pull vacuum for 5-10 min. Seal the desiccator and leave the wafers under vacuum for a period of $12-16 \mathrm{~h}$.

CAUTION: The silane is toxic and should not be inhaled. Take care to work in a fume hood and to wear nitrile gloves when handling the silane. This includes placing the vacuum pump in the fume hood when pulling vacuum on the desiccator.

18. Release the vacuum from the desiccator and remove the silanized wafers. Rinse with water and use a steam of $\mathrm{N}_{2}$ to dry the wafers. At this point the wafers can be placed in storage until required.

\section{Microfluidic device fabrication}

NOTE: The soft-lithography process used to fabricate PDMS based multilayer microfluidic devices can be separated into three distinct steps: 1) The PDMS preparation of both the flow and control layers, 2) The alignment and bonding of the two PDMS layers, 3) The completion of the device.

1. PDMS preparation

1. Prepare two PDMS precursor solutions by combining the base and curing agents in a plastic beaker and using a mixing rod to stir the two components until fully mixed. The control layer requires $20 \mathrm{~g}$ of base agent and $1 \mathrm{~g}$ of curing agent (20:1 ratio). The flow layer requires $40 \mathrm{~g}$ of the base agent and $8 \mathrm{~g}$ of the curing agent (5:1 ratio). Degas the solutions in a desiccator.

2. Place the flow layer wafer in a Petri dish and pour the 5:1 ratio PDMS mixture over the wafer. Degas the PDMS for 30 min to remove air bubbles.

3. Spin coat the control layer wafer (prepared using the negative SU8 3050photoresist) with 20:1 ratio PDMS. Pour 5-10 mL of the PDMS onto the center of the wafer and run the following spin protocol (retain the left over PDMS for later use): spin at $500 \mathrm{rpm}$ for $15 \mathrm{~s}$ $(100 \mathrm{rpm} / \mathrm{s})$, increase the spin speed to $1450 \mathrm{rpm}(300 \mathrm{rpm} / \mathrm{s})$ for $45 \mathrm{~s}$, and then decelerate the wafer to $0 \mathrm{rpm}(200 \mathrm{rpm} / \mathrm{s})$.

4. To ensure a homogeneous PDMS film thickness, place the PDMS coated wafer on a level surface in a closed Petri dish (to avoid dust contamination). Let the wafer sit for $30 \mathrm{~min}$.

5. Remove the flow layer from the desiccator and place both the flow and control layers in an oven $\left(80^{\circ} \mathrm{C}\right)$. Cure both layers for $28-30$ min and remove when the PDMS is malleable enough to manipulate, whilst remaining slightly sticky. Immediately proceed with the alignment process.

\section{Alignment and bonding}

1. Using a scalpel, remove each of the four devices from the PDMS on the flow layer wafer. Upon removing the PDMS layer from the silicon wafer, immediately cover the feature side with scotch tape to avoid dust particle contamination.

2. Roughly align the flow layer blocks on the control layer by eye, placing the feature side of the device into contact with the control layer PDMS. Subsequently, make fine adjustments to the position of each of the flow layer blocks to align the channels of the flow layer with the control layer channels, using a stereo microscope to aid in the visualization of the adjustments.

3. Apply pressure to remove air pockets between the two PDMS layers. Pour the remaining 20:1 ratio PDMS saved earlier around the aligned flow layer blocks. Place $100 \mathrm{~g}$ weights on each of the devices to ensure sufficient contact during the bonding process.

4. Return the aligned devices (including the weights) to the $80^{\circ} \mathrm{C}$ oven and leave them to bond for at least $1.5 \mathrm{~h}$ and no longer than $6 \mathrm{~h}$. 
3. Finishing the Device

1. Remove the wafer from the oven and extract each of the individual devices from the control layer wafer, covering the feature side of each device with scotch tape.

2. Iteratively, punch a single hole for each of the 9 flow layer inlets, 24 control layer channel inlets, and the single flow layer outlet of each device. Punch the device with the feature side facing up, using a camera to ensure holes are punched within the feature boundaries

3. For each device, clean a single microscope slide with isopropanol and acetone and dry the slides under a stream of $\mathrm{N}_{2}$. Subsequently, place the microscope slides on a hot plate set to $150^{\circ} \mathrm{C}$ for $15 \mathrm{~min}$.

4. Use oxygen plasma ashing to bond the PDMS devices to the glass slides, applying an ashing power of $50 \mathrm{~W}$ for $45 \mathrm{~s}$. Ensure that the feature side of the device is facing upwards when ashing. Once complete, place the device feature side down on the glass slide, applying pressure to remove trapped gas between the surfaces.

5. Place the bonded devices onto a hot plate set to $110^{\circ} \mathrm{C}$ for $1 \mathrm{~h}$. Weights can be placed on top of the devices to improve adhesion of the device.

\section{Hardware setup}

NOTE: To achieve control over the microfluidic chips, numerous pieces of hardware need to be installed and connected with one another. Three distinct groups of hardware are required: 1) Pneumatic control system for the control channels, 2) A pneumatic pressure regulator to control flow of the reaction reagents within the device, and 3) A cooling system to cool the IVTT reaction solution prior to injection into the microfluidic device. An overview of the hardware setup is provided in Figure 1. It should be noted that the protocols provided here attempt to be as general as possible, however certain specific pieces of equipment used throughout our research are referenced. All hardware can be replaced by alternatives able to perform the same function. In such cases, the protocols here can be used to outline the general steps needed to set up the system and the requirements of each of the components. Alternative hardware setups are presented by Brower et al. ${ }^{48}$ and White and Streets ${ }^{49}$.

1. Pneumatic control system (see Figure 2)

1. Using the manufacturers' protocol, establish a TCP connection between the fieldbus controller and the user workstation. Use control software (provided as supplementary files) to compose MODBUS commands which are sent via the TCP connection to controller, and actuate the solenoid valves.

2. Expand the fieldbus controller with eight 4-channel digital output modules, one for each solenoid valve being used. Each solenoid valve presides over a connecting pin. Connect the positive wire to one of the four positive outputs on one of the digital output modules whilst connecting the negative wire to one of the ground ports of the digital output modules.

NOTE: To get the system to work correctly, the solenoids should be connected systematically, with the first solenoid being connected to the first output port, the second solenoid to the second output port and so on. In our system, two valve arrays are used with 8 and 22 solenoid valves respectively. Output ports 1-8 connect to the 8-valve array, and output ports 9-30 connect to the 22-valve array.

3. Connect both valve arrays to a compressed air source using 1/4" tubing. Use pressure regulators to set the pressure of the 22 -valve array to 3 bar, and the 8 -valve array to 1 bar.

2. Flow pressure regulation (see Figure 3)

NOTE: To flow fluids through the flow layer of the microfluidic device, a commercially available 4-port pressure regulator is used. The output pressure of each port is regulated via software provided with the pressure controller. Connect the pressure regulator to a computer using the supplied USB-connector.

1. Connect the pressure regulator to a compressed air source, ensuring that the supplied pressure does not exceed the maximum pressure permitted by the regulator.

2. Connect a male Luer to $3 / 32$ " barb connector to each of the four female Luer lock output ports of the pressure regulator. Connect a length of soft tubing (OD: $3 \mathrm{~mm}$, ID: $1 \mathrm{~mm}$, L: $10 \mathrm{~cm}$ ) to the barb.

3. Connect a second male Luer to $3 / 32$ " barb connector to the open end of the soft tubing and attach this to the fluid reservoir connector ports.

4. Use the provided software to set the desired pressure of each outlet of the flow regulator, pressurising the reagents stored within the reservoirs to result in the flow of the reagents into the microfluidic device. The connection of the reservoirs to the microfluidic device will be discussed in the section 4.2 .

3. Off-Chip cooling setup (see Figure 4)

1. Use PVC tubing (OD: $10 \mathrm{~mm}$, ID: $6 \mathrm{~mm}$ ) to connect the water-cooling system to the cold-plate water block using compression fittings. Fill the fluid reservoir of the water-cooling system with a coolant and gently tilt the unit to displace any trapped air, continuously adding coolant to the reservoir to ensure it remains full. When all the gas is removed from the system, fill the reservoir to approximately $90-95 \%$ of its maximal volume.

2. Coil PTFE tubing (OD: 0.042", ID: 0.022") onto the cold face of the Peltier element and secure this with tape. Ensure that one end of the PTFE tubing is connected to the reservoirs of the flow layer pressure control system (as described in section 4.3). The other end of the PTFE tubing should protrude no more than $1 \mathrm{~cm}$ from the Peltier surface. Insert a $5-10 \mathrm{~cm}$ length of PEEK tubing (OD: $0.794 \mathrm{~mm}$, ID: $0.127 \mathrm{~mm}$ ) into the protruding end of the PTFE tubing. The filling of the tubing and connection to the microfluidic device is further explained in section 4.3 .

3. Place the hot face of the Peltier element onto the cold-plate of the water block, applying sufficient thermal compound to the two faces. Ensure that the tubing, Peltier element, and cooling block are in direct contact with one another at all times.

4. Connect the Peltier element to the temperature controller (via a serial bus connector), such that the voltage supplied to the Peltier can be regulated. Securely place a thermistor on the Peltier surface, connecting the output to the temperature controller. After turning on the water cooler, adapt the voltage supplied to the Peltier until the temperature is stable at $4{ }^{\circ} \mathrm{C}$.

NOTE: With this setup, the Peltier temperature is controlled manually by adapting the supplied voltage, while the thermistor serves only to monitor the temperature. 


\section{Preparing an experiment}

NOTE: Prior to starting an experiment, the microfluidic device must be prepared, and the reaction reagents must be inserted into the correct tubing for injection into the device. This section will discuss: 1) The connection of control channel tubing to the device, 2) The connection of uncooled inflow reagents to the device, and 3) The connection of cooled inflow reagents to the device.

1. Connecting the control channel tubing

1. For each of the control channels of the microfluidic device, cut a length of tubing (OD: 0.06", ID: 0.02 "). At one end, insert the pin of a 23 G, 1/2" Luer stub and at the other insert a stainless steel connecting pin (OD: $0.65 \mathrm{~mm}$, ID: $0.35 \mathrm{~mm}, \mathrm{~L}: 8 \mathrm{~mm}$ ).

2. Connect the Luer stub to a male Luer to $3 / 32$ " barb nylon connector. Insert the barb of the connector into a length of polyurethane tubing (OD: $4 \mathrm{~mm}$, ID: $2.5 \mathrm{~mm}$ ). Insert this polyurethane tubing directly into one of the solenoid valves.

3. Attach a $23 \mathrm{G}, 1 / 2$ " Luer stub to a syringe and insert this into a short (3-4 cm) piece of tubing (OD: 0.06", ID: 0.02"). Place the open end of this tubing into a reservoir of ultrapure water and fill the syringe with ultrapure water.

4. Number each control channel of the microfluidic device as shown in Figure 5. For each channel (excluding control channels 1 through 3 , which are not filled with water), find the corresponding tubing (connected to the solenoid valves) and insert a metal pin into the open end of the tubing attached to the syringe. Inject water into the control channel tubing until half the length has been filled.

5. Disconnect the tubing from the syringe and insert the stainless-steel connector pin into the corresponding hole of the microfluidic device. Repeat for all control channels.

6. Use the control interface to open all the solenoid valves. This will pressurize the fluid within the control channel tubing, forcing it into the microfluidic device and closing all the membrane based valves within the device. An example of open and closed membranes within the device are given in Figure 6.

2. Connecting uncooled reagents to the device

1. For each of the uncooled reagents, cut a length of tubing (OD: 0.06 ", ID: 0.02 ") to connect the reservoir outlet to the microfluidic device inlets.

2. Take one end of the tubing and insert this into the reservoir, ensuring that the tubing reaches the base of the reservoir. The reservoir tubing outlet should be tightened such that an air tight seal is achieved. Insert a stainless-steel connection pin (OD: $0.65 \mathrm{~mm}$, ID: 0.35 $\mathrm{mm}, \mathrm{L}: 8 \mathrm{~mm}$ ) into the open end of the tubing.

3. Attach a $23 \mathrm{G}, 1 / 2$ " Luer stub to the end of a small (1 mL) syringe. Add a short length of tubing (OD: 0.06", ID: 0.02 ") to the Luer stub. Placing the end of the tubing into the desired reagent solution, fill the syringe with the reagent.

4. Insert the stainless-steel connector pin into the polyurethane tubing connected to the syringe and fill the tubing with the reagent. When using small reaction volumes, the reagent will not enter the reservoir, and the tubing itself will act as the reservoir. Disconnect the syringe and insert the connector pin into one of the flow layer inlet holes of the microfluidic device.

5. Apply pressure to each of the reservoirs using the pressure regulator software to force the reagents into the microfluidic device.

3. Connecting cooled fluids to the microfluidic device

1. Ensure that the water cooler and Peltier element have been turned on, with the surface temperature of the Peltier set to $4{ }^{\circ} \mathrm{C}$. Mount the cooling setup as close to the microfluidic device as possible, minimising the uncooled volume between the Peltier and the device inlet.

2. Connect the open end of the PTFE tubing to tubing connected to one of the fluid reservoirs (as described in section 4.2) using a stainless-steel connector pin (OD: $0.65 \mathrm{~mm}$, ID: $0.35 \mathrm{~mm}, \mathrm{~L}: 8 \mathrm{~mm}$ ).

3. Connect a small syringe ( $1 \mathrm{~mL})$ to a Luer stub (23 G, 1/2") with a short length of tubing (OD: 0.06 ", ID: 0.02 ") attached to the end. Fill the syringe with the to-be-cooled reagent (here, the IVTT reaction solution).

4. Connect the PEEK tubing to the syringe via the connective tubing and apply constant pressure to the syringe, forcing the reagent through the PEEK tubing and into the PTFE tubing. Disconnect the PEEK tubing from the syringe and insert it directly into one of the flow channel inlets of the microfluidic device. When pressure is applied via the pressure regulator software the cooled reagent will be forced into the microfluidic device.

\section{Experimentation}

NOTE: Prior to performing experiments all the hardware and tubing connections detailed in protocols sections 3 and 4 should be completed, and all the reagents should be connected to the device. The experimental procedure can then be divided into four distinct parts: 1) The loading of the microfluidic device, 2) Preparing the microscope, 3) The calibration of the device, and 4) Performing the experiment. The custom virtual control interface (see Figure 7) used throughout this research is provided as a supplementary resource via the Materials list)

1. Loading the microfluidic device

1. Place the microfluidic device, with all control and flow layer tubing attached into the microscope stage and close any openings on the incubator. Set the ambient temperature of the incubator to $29^{\circ} \mathrm{C}$. Ensure that the cooling system has been turned on and is set to $4{ }^{\circ} \mathrm{C}$ prior to initiating the experiment.

2. Ensure all the flow and control channels are pressurized. Set the pressure of control channels 1-3 at 1 bar, and pressurize control channels $9-29$ at 3 bar. The reagents require a pressure of between 20 and 100 mbar to be applied to the fluid reservoirs using the pressure regulator software.

3. Remove air from the microfluidic device using one of the reagents. Close the outlet of the device (pressurize control channel 29) and simultaneously depressurize control channels 1-3, and 15-28. Then selectively depressurize the control channels of the multiplexer to allow the selected reagent to flow into the device. Use the microscope to monitor the removal of air.

NOTE: In the case that reagents are not loading into the device correctly, or that the air bubbles are not being removed, the pressure can be increased up to 350 mbar. 
4. Ensure that all reagents flow correctly, without introducing air - using the Flush function in the control software. Monitoring of the fluid flow can be simplified by first loading a fluorophore and monitoring its displacement by individual reagents.

2. Preparing the microscope

1. Using the microscope, locate any points of interest (a single point within each reactor is sufficient) within the microfluidic device, and store the coordinates thereof. These points will be imaged during the calibration and experimental processes.

NOTE: During the calibration and experimental procedures included within the control software, the microscope is instructed to periodically capture images at the previously stored coordinates. To achieve this, the control software communicates with the microscope software, informing it to record new images. This communication is unique to each microscope setup, and as such this functionality has been modified within the provided software interface. Provided is a dummy executable, which can be modified by the end-user for compatibility with their own microscope system.

3. Calibrating the microfluidic device

1. Determine the fluid volume displaced from each reactor during a single inflow step (pump sequence provided by peristaltically actuating control channels 15-17, comprising 6 MODBUS commands executed sequentially), by executing the calibration protocol provided in the software package.

2. Set the following data fields within the control software: Elution Buffer Channel, Fluorophore Channel, Number of Dilution Cycles (default is 10 dilutions), Number of Inflow Steps (default is 15 steps), Number of Mixing Cycles (default is 4 cycles), and Time between Mixing Cycles (default is 0 seconds). Initiate the calibration by pressing Perform Calibration Experiment. NOTE: During the calibration process, all reactors are filled with a fluorophore and images are recorded. Subsequently, a series of dilutions are performed where an eluent is metered into the reactors (based on the set number of inflow steps), thus displacing the fluorophore. After thoroughly mixing, new images are taken. This process repeats for the set Number of Dilution Cycles.

3. Upon completion of the calibration, follow the steps presented by the control software, completing the analysis of the calibration experiment.

NOTE: The analysis will provide users with the Refresh Ratio for each reactor based on the fluorescence decrease recorded during each Dilution Cycle. This value indicates the fraction of the reactor volume displaced by the set Number of Inflow Steps. In turn, this value will be used during experiments to determine how many inflow steps are required to displace a specific reactor volume.

4. Performing the Experiment

1. Set the required values for the desired experiment within the virtual control interface. Critical is the Refresh Fraction [\%] which determines the reactor volume displaced per experimental cycle and should be set between 15 and $40 \%$.

NOTE: The specific experimental protocol will determine which fields of the control interface should be set prior to the initiating the experiment. Some minor coding will be required to adapt the control interface for novel experiments.

2. Initiate the experimental protocol by pressing the Perform Experiment button in the control interface.

NOTE: Unaltered, the provided software initiates a simple protein expression. Reactors 1 and 8 are utilized as controls, while reactors

2-7 house identical experiments. Here, $75 \%$ of the reactor volume comprises IVTT solution, and $25 \%$ is either ultrapure water or a 2.5 nM linear DNA solution. Dilutions occur every 15 minutes, with $30 \%$ of the reactor volume being displaced per dilution. Images are recorded at the end of each dilution cycle.

\section{Data analysis}

NOTE: Scripts have been provided for the analysis of the images (see supplementary files or the Table of Materials), making use of the 'bfopen' analysis package, which is required for the reviewing of '.nd2' files (provided by our microscope setup).

1. Run the analysis script 'calibrationScript.m' and once prompted select the desired '.nd2' file. A single reactor image will be shown with which the correct image intensity can be determined. Use the slider to optimize the image intensity such that the edges of the microfluidic channel are clearly visible.

2. An image of the reactor will be shown. Within this image, select an area inside the reactor channel of which the fluorescence intensity should be determined.

NOTE: The fluorescence intensity of each reactor, for each recorded image will be determined with the results displayed in a simple plot, allowing the visualisation of the results.

\section{Representative Results}

To demonstrate the effectiveness of the multilayer microfluidic platform for the conduction of IVTT experiments, the described setup was used to express the deGFP protein. The experiment was conducted in a commercially available ${ }^{30}$ IVTT reaction mixture - comprising all the necessary transcription and translation componentry - supplemented with reaction substrates and DNA templates. Experiments were conducted at a temperature of $29^{\circ} \mathrm{C}$; a temperature found to be optimal for the IVTT expression of proteins. 
The microfluidic device possesses nine unique inlets, of which four were utilized during this experiment. The first contained the commercially obtained IVTT reaction mixture. The IVTT reaction mixture accommodates all the components required to successfully express proteins however, purified GamS was added to the reaction mixture - at a final concentration of $1.3 \mu \mathrm{M}$ - prior to loading into the microfluidic device. The addition of the GamS protein serves to minimize the degradation of linear DNA species when performing the experiments. Crucially, the IVTT mixture was injected into polytetrafluoroethylene (PTFE) tubing coiled onto a Peltier element with a surface temperature of $4{ }^{\circ} \mathrm{C}$ to cool the solution prior to the injection thereof into the microfluidic device; preventing the degradation of the reaction solution prior to its use. Microbore polyether ether ketone (PEEK) tubing was used to connect the PTFE tubing leaving the Peltier element surface with the microfluidic device, reducing the volume of the IVTT reaction mixture not being cooled. The second solution inserted into the device contained the linear DNA template coding for the deGFP - dissolved in ultrapure water - at a concentration of $10 \mathrm{nM}$. The third solution, ultrapure water, served multiple purposes during the experimental procedures. Primarily, the ultrapure water was used to ensure that the displaced volume per dilution was equal for all reactors, acting as a replacement for DNA in the control reactions. Additionally, ultrapure water was also used to dilute the fluorophore during the device calibration and to flush the dead volume of the device when switching between reagents. The final solution inserted into the device was a purified FITC-dextran solution $(25 \mu \mathrm{M})$ required to perform the initial device calibration. The DNA, water, and fluorophore solutions were injected into tubing (0.02" ID, 0.06" OD) which could subsequently be inserted into one of the inflow channels of the microfluidic device as per Section 4.2 of the protocols. As such, these solutions were stored at $29^{\circ} \mathrm{C}$ for the entirety of the experiments.

The actuation of the control channels of the microfluidic device is achieved via custom control software where each of the control channels can be individually actuated. The execution of prolonged IVTT reactions cannot be achieved via this manual process and requires the use of automated protocols incorporated within the control software. When preparing a microfluidic device for experiments, similar automated protocols can be utilized to execute a number of useful processes: the flushing of the device dead volume with a new reagent, the mixing of the reagents within the ring reactor, and the loading of a new reagent into the reactor whilst displacing an equal volume of the current solution. In addition, two complex process are available: the conduction of a device calibration, and the execution of a prolonged cell-free protein expression. All of the aforementioned processes can be easily executed from the main interface, alongside the ability to configure multiple parameters to vary specific process settings such as the inflow channel, inflow volume, and mixing duration.

Due to fluctuations in pressure and imperfections during microfluidic device fabrication, the volume of fluid displaced during a single injection cycle can vary between devices. As such, prior to performing IVTT experiments, the displaced reactor volume per injection cycle (Refresh Fraction) was determined. This calibration requires the filling of all eight reactors with a fluorescent reference solution. In this case, a purified FITC-dextran solution $(25 \mu \mathrm{M})$ was used. Subsequently, the reactors are diluted 10 times with ultrapure water. By measuring the decrease in fluorescence per dilution cycle for each reactor, the volume of fluid displaced during a single injection cycle was determined. Within the control software, this value (the Refresh Ratio) was recorded for use during the IVTT experiment. Crucially, to account for variations in the flow rate across the device, as well as discrepancies in the individual reactor volumes, the Refresh Ratio is determined and stored for each individual reactor. The sequence of filling and diluting the reactors was conducted automatically using the Perform Calibration program which forms part of the control software. The results of the calibration experiment are shown in Figure 8.

The most complex pre-programmed process executes a long-duration IVTT experiment, allowing users to initiate the experiment and subsequently allow it to operate unattended until completion. Throughout the experiment, reactors 1 and 5 were used as blanks, with only water being added to the reactors during dilutions. Reactors 2 and 6 were utilized as negative controls and contained only IVTT reaction solution and ultrapure water. The remaining reactors $(3,4,7$, and 8$)$ contained the IVTT reaction solutions and $2.5 \mathrm{nM}$ of linear DNA coding for the deGFP gene. Initialisation of the reactors is achieved by fully filling all the reactors (excluding 1 and 5 ) with the IVTT reaction solution, before $25 \%$ of the reactor volume was displaced with ultrapure water. Hereafter, the periodic injection of reagents into the reactors was initiated. The experiment was conducted such that new reagents were injected into the reactors every 14.7 minutes, with $30 \%$ of the reactor volume being displaced during each dilution cycle. The composition of each injection was such that $75 \%$ of the injected fluid comprised fresh IVTT solution, whilst the remaining $25 \%$ consisted of either DNA or ultrapure water. Following each injection of new reagents the reactors were continuously mixed, after which a fluorescence image of each reactor was recorded using the microscope. The reaction was subsequently allowed to run continuously for 68 cycles, resulting in an experimental duration of $16.5 \mathrm{~h}$. The results of this experiment are given in Figure 9.

When performing prolonged IVTT experiments, there are two main causes for the failure of a reaction; the introduction of air into the microfluidic device or the degradation of the IVTT reaction solution. The occurrence of air within the microfluidic device is most often the direct result of small air bubbles existing in the inflow solutions, which are subsequently injected into the microfluidic device. Upon entering the device, the presence of air inhibits the proper flow of fluids, whereby the reactions are no longer periodically refreshed leading to the formation of batch reactions within the reactor rings. In some cases, the air is slowly removed from the device by the repeated flushing of reagents, after which the reaction continues as intended (as shown in Figure 9). In other cases the air remains trapped, and can only be removed by aborting the experiment and subsequently applying continuous (high) pressure to the flow layer of the microfluidic device, analogous to the filling process described in Section 5.1 of the protocols. During our experiments the cell lysate is stored in PTFE tubing on a Peltier element cooled to $4{ }^{\circ} \mathrm{C}$. Both measures aid in limiting the degradation of the IVTT reaction solution over time, with the inert PTFE tubing ensuring limited interaction between the tubing and the reaction solution and the cold temperatures preserving the functional (bio)molecular componentry required to perform IVTT. Should degradation of the reaction solution occur - as the result of insufficient cooling or undesired interactions between the reaction solution and the storage environment - then this will exhibit itself experimentally as a gradual reduction of protein expression over time. Once degraded, the IVTT reaction solution cannot be recovered and a new experiment should be prepared. 
A
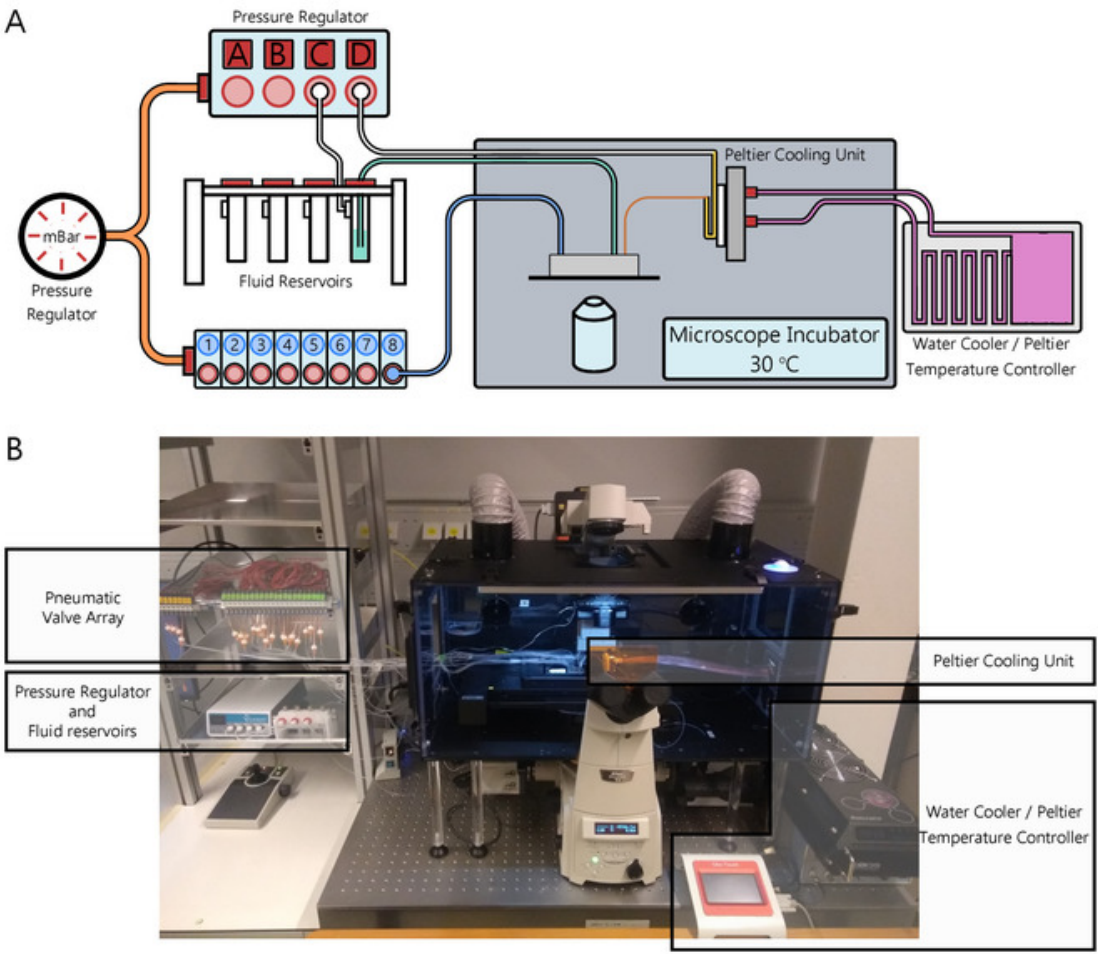

Figure 1. The hardware setup required to perform continuous IVTT reactions. A) Schematic of the hardware setup. B) Photograph of the setup used throughout this manuscript. The implementation of a multilayer microfluidic device for continuous IVTT reactions requires an extensive hardware setup to regulate flow pressure, actuate control channels, heat and cool reactions and reagents, store fluids, and image the device during experiments. Experiments are performed at temperatures of $30^{\circ} \mathrm{C}$, which is achieved by placing the microscope within an incubator set to this temperature. To prevent deterioration of the IVTT reaction solution, it is stored within PTFE tubing coiled over the cold face of a Peltier element. The temperature of the Peltier element is set to $4{ }^{\circ} \mathrm{C}$, with a water cooler and water block being used to maintain this temperature. Reagents which do not require cooling, are stored in fluid reservoirs outside of the microscope incubator. Constant pressure is applied to these reservoirs by a computer controlled pressure regulator. In this manner, the fluids are forced through the outlet tubing of the reservoirs, which connect directly to the inflow channels of the microfluidic device. Each of the control channels of the microfluidic device is connected to a pneumatic valve. The entire valve array is under constant pressure. Opening the valve, allows for pressurisation of the fluid within the tubing connecting the pneumatic valve to the control channel of the microfluidic device, thus opening and closing the PDMS membranes found within the microfluidic device. The pneumatic valves are opened and closed via a user interface which commands a fieldbus controller (not shown) to open and close specific pneumatic valves. Figure adapted from Yelleswarapu et al. ${ }^{22}$. Please click here to view a larger version of this figure. 


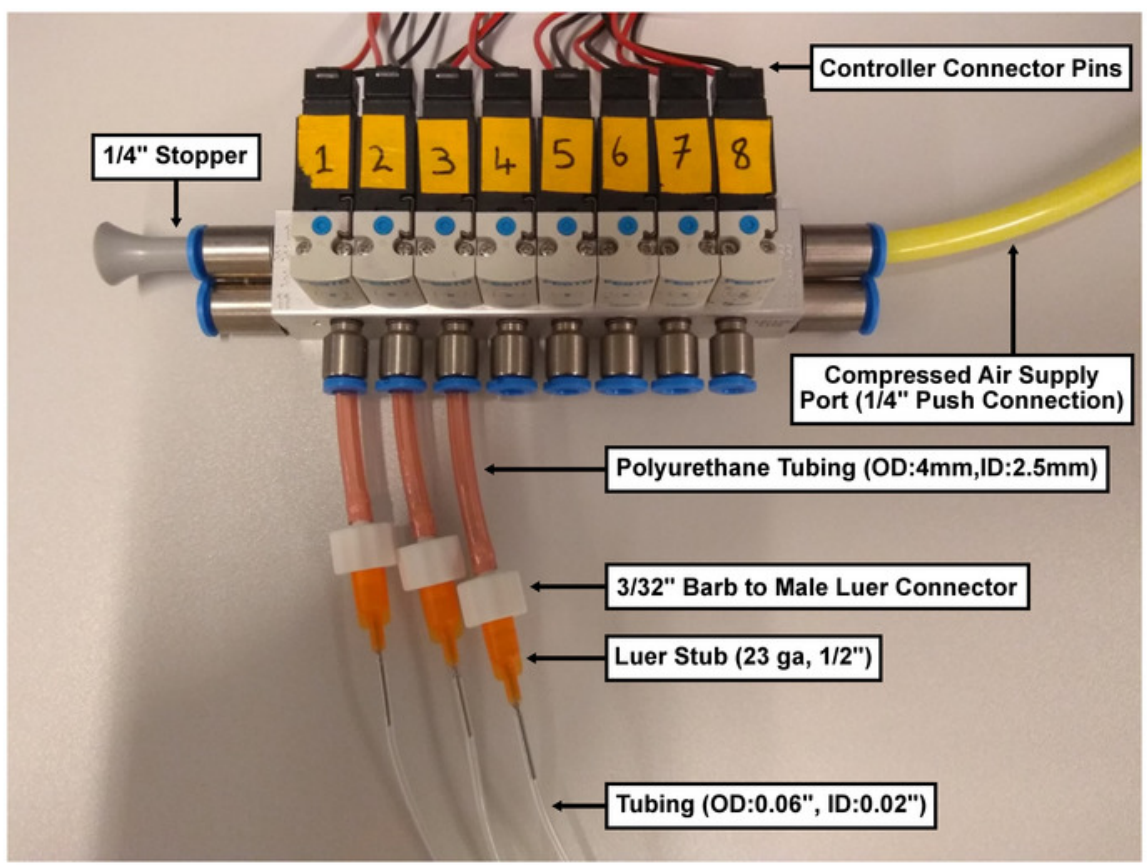

Figure 2. Overview of the pneumatic valve setup and control channel connection. An 8-valve array is shown with three control channel connections fitted to valves 1,2 , and 3 . Compressed air can be supplied to the valve array via 1/4" tubing. For the actuations of control channels two pressures are used: 1 bar for the lower pressure control channels (1, 2, and 3 ) and 3 bar for the higher pressure control channels ( 9 through 30 , not shown here). The tubing can be filled with ultrapure water and inserted into one of the control channel inlets using a stainless steel connector pin. Please click here to view a larger version of this figure.

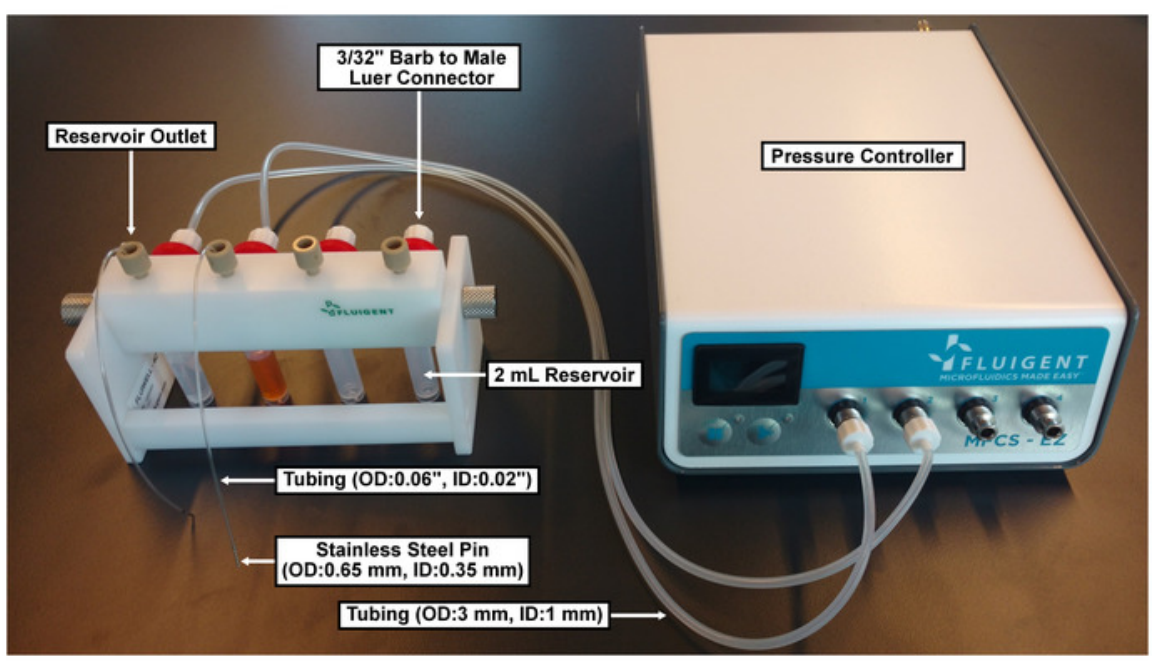

Figure 3. Overview of the commercial flow pressure regulator and reservoir system. A commercially available pressure regulator is used to inject fluids into the flow layer of the multilayer microfluidic device. Connecting the pressure controller to a computer allows for modulation of the pressure used to perform the fluid injections. Reagents can be stored in a fluid reservoir, which is directly connected to the pressure regulator. The application of pressure to the reservoir forces the fluid out of the reservoir via the outlet tubing. This outlet tubing can be connected directly to one of the fluid inlets of the microfluidic device using a stainless steel connector pin. In the event that the reagent volume is unable to reach the fluid reservoir, the outlet tubing acts as a reservoir for the reagent. Please click here to view a larger version of this figure. 


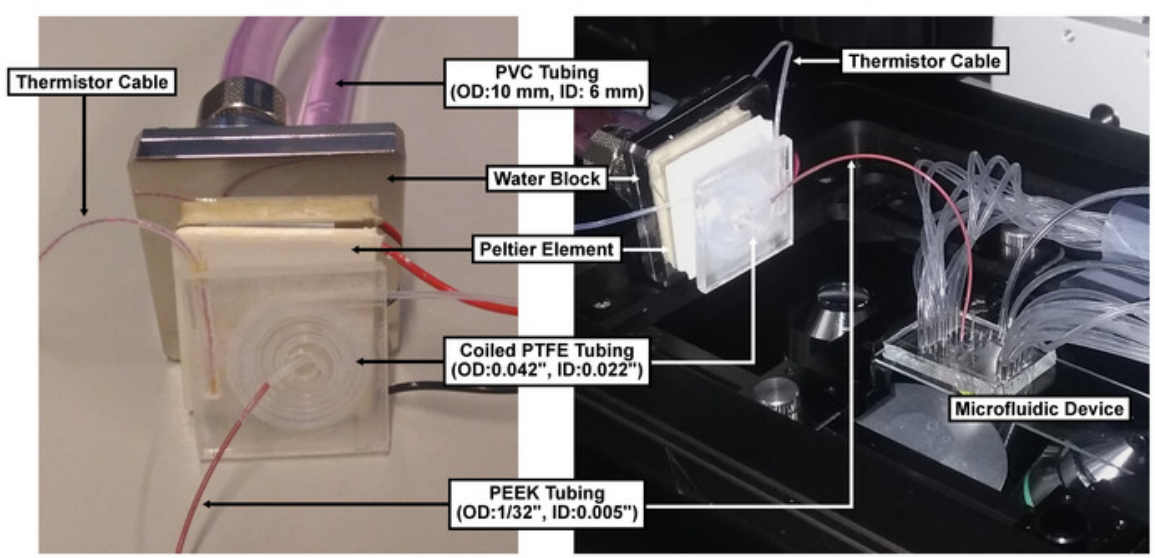

Figure 4. Overview of the cooling system used to cool reaction reagents. (Left) Isolated cooling setup and (Right) Cooling setup placed within the microscope and connected to the microfluidic device. A Peltier element is used to cool the IVTT reaction solution prior to injection into the microfluidic device. The reagent is stored within PTFE tubing coiled over the cold-face of the Peltier element. A length of PEEK tubing is used to transfer the cooled fluid to the microfluidic device, with the small internal diameter (0.005") minimizing the reagent volume no longer being cooled. Alongside the coiled PTFE tubing, a thermistor is placed, allowing for real-time temperature monitoring on the surface of the Peltier element. The voltage applied to the Peltier is set such that the surface temperature of the Peltier remains between $0{ }^{\circ} \mathrm{C}$ and $4{ }^{\circ} \mathrm{C}$. To remove excess heat produced by the Peltier element, the hot-face of the Peltier is placed against a water cooled block, with the addition of silicone free heat sink grease ensuring optimal heat transfer between the two faces. Please click here to view a larger version of this figure.

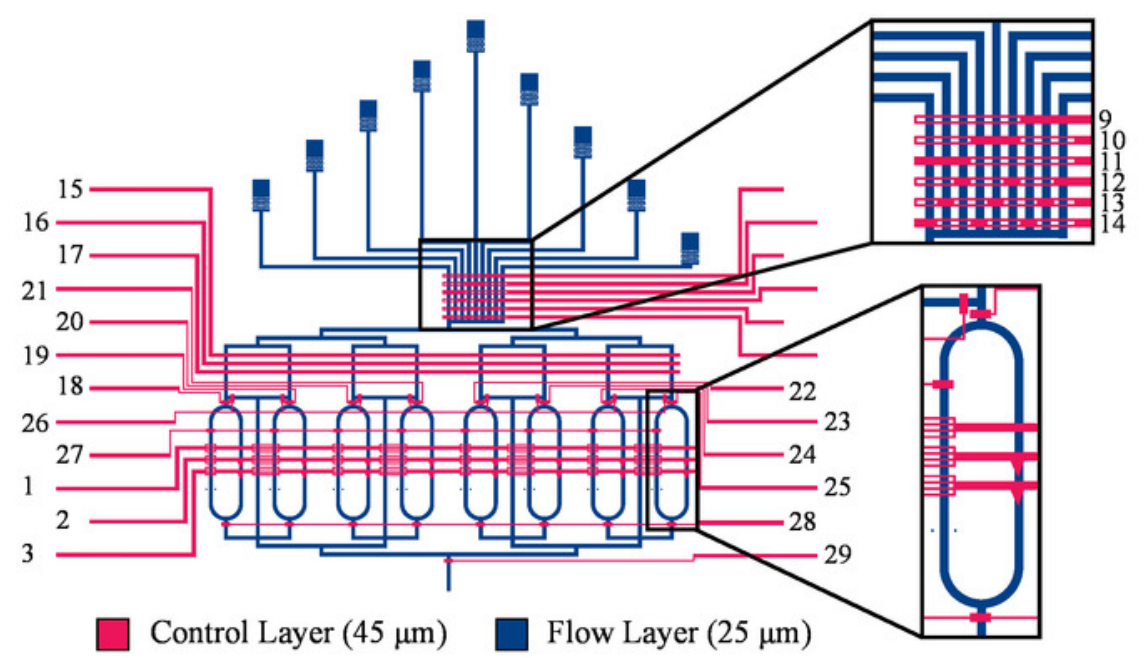

Figure 5. Overview of the microfluidic device design. The microfluidic flow reactor for continuous IVTT reactions consists of eight reactor rings, each with a volume of $10.7 \mathrm{~nL}$. Nine inlets allow for the inflow of nine unique reaction solutions into the device. 24 control channels regulate the flow of fluids within the device. Control channels 9 through 14 form a multiplexer. These control channels should be pressurized at all times to inhibit fluid flow into the device. Depressurisation of two control channels simultaneously allows for the inflow of a single reagent. Control channels 15,16 , and 17 are used to peristaltically pump the reagents into the device in a controlled manner. Control channels 18 through 25 each control the inlet of one of the eight reactors found within the device. Control channel 26 can close the flush channel, thus forcing fluid into the reactors. Control channel 27 aids in the homogeneous filling of the reactors. Control channels 28 and 29 regulate the ring reactor outlets and the only device outlet respectively. Finally, control channels 1, 2, and 3 are used to peristaltically pump the fluid within the ring reactors, resulting in mixing of the reagents. The design of this microfluidic device and the figure are both adapted from Neiderholtmeyer et al. ${ }^{29}$. Please click here to view a larger version of this figure. 

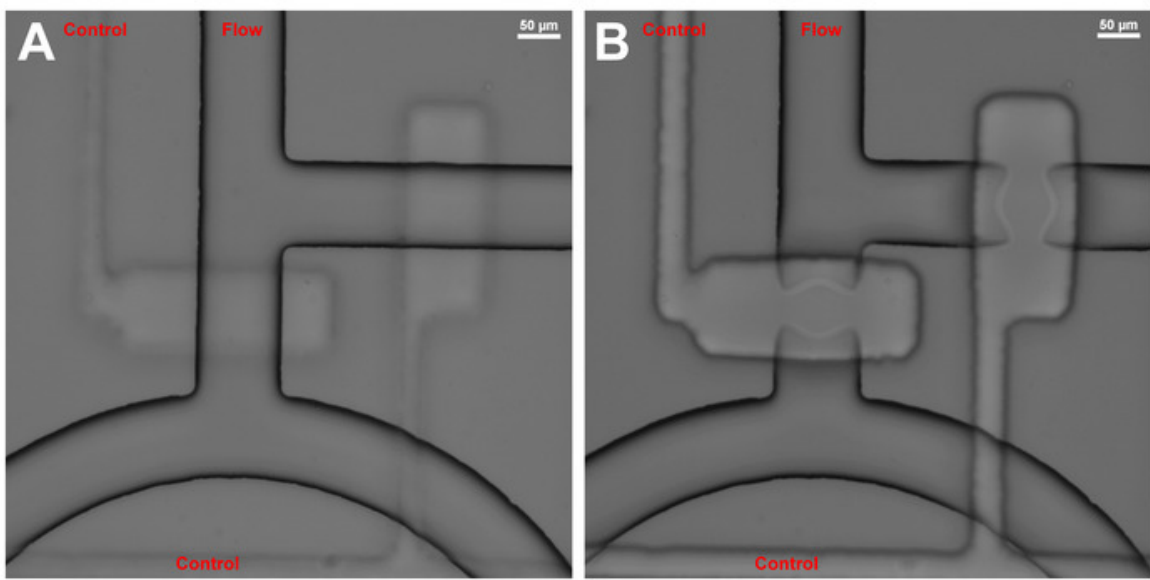

Figure 6. Membrane based valve within the microfluidic device. A) Flow channel within the microfluidic device. Two control channels can be seen in the background. These channels are not pressurized and as such the valves are open (fluid can flow). B) The two control channels intersecting the flow layer channels have been pressurized, closing the valves (i.e., fluid flow is impeded). Upon pressurization of the control channels, the thin PDMS membrane separating the flow and control layer channels is deflected upwards (the control layer lies beneath the flow layer) which closes the flow layer channel. The rounding of the flow layer channel is critical in ensuring that the deflected membrane fully closes the flow channel. Please click here to view a larger version of this figure.

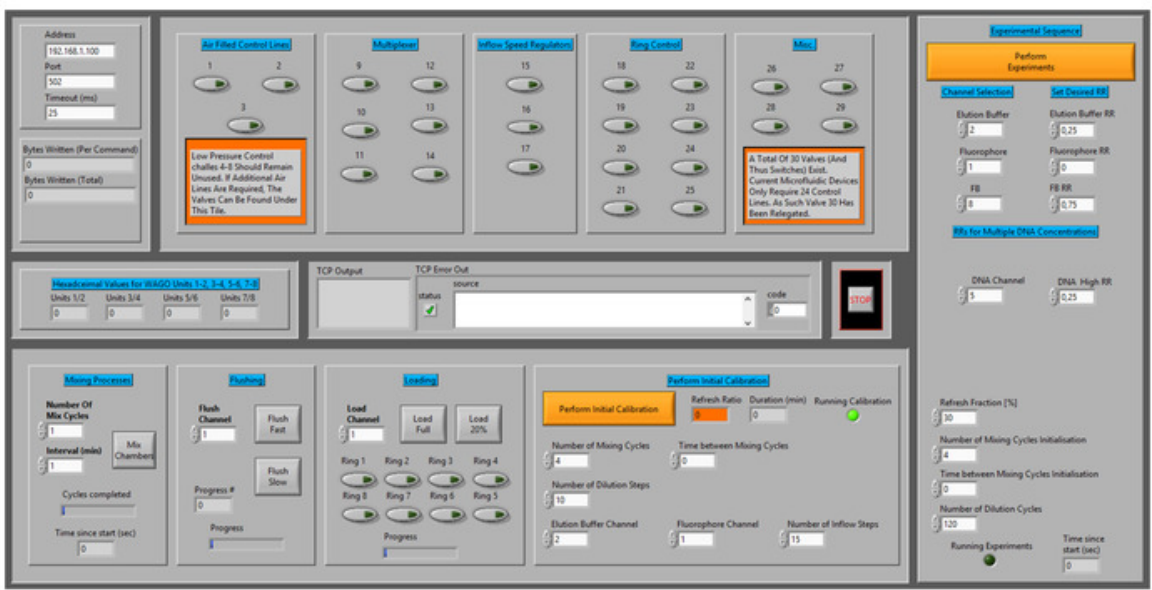

Figure 7. User interface used to control microfluidic device. Throughout this research, a custom control interface has been used to control the flow of fluids within the microfluidic devices. The interface allows users to individually actuate each of the control channels (numbered 1-3 and 9-29), or to execute elaborate protocols resulting in the flushing and loading of reagents, the calibration of the microfluidic device, and the execution of experiments. Please click here to view a larger version of this figure. 

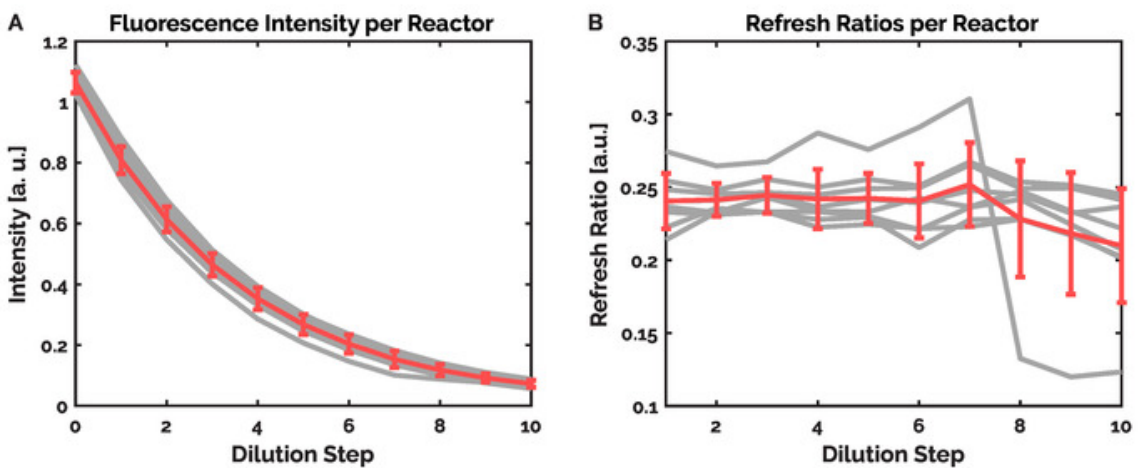

Figure 8. Results of a calibration experiment. During a calibration experiment, the reactors are filled with a fluorophore ( $25 \mu \mathrm{M}$ FITC-Dextran) after which the fluorescence intensity is recorded. Subsequently, a series of dilutions follow, where a set number of inflow steps (15) are used to inject ultrapure water into the reactors. After each dilution, the reagents are mixed and the fluorescence is measured. The decrease in the fluorescence intensity per dilution reveals the volume of the reactor ring displaced for the set number of inflow steps; a value termed the Refresh Ratio. A) The average intensity and standard deviation of all eight reactors is shown in red, with the individual intensity traces shown in grey. B) The average Refresh Ratio and standard deviation is shown for each dilution step in red. The individual Refresh Ratios of each individual reactor are shown in grey. It can be seen that seven of the eight reactors show very similar behavior, however one reactor shows fluctuations in the Refresh Ratio after the seventh dilution cycle. This highlights the need for unique Refresh Ratios for each of the reactors, as opposed to using an average Refresh Ratio for the injection of reagents into the reactors. Please click here to view a larger version of this figure.

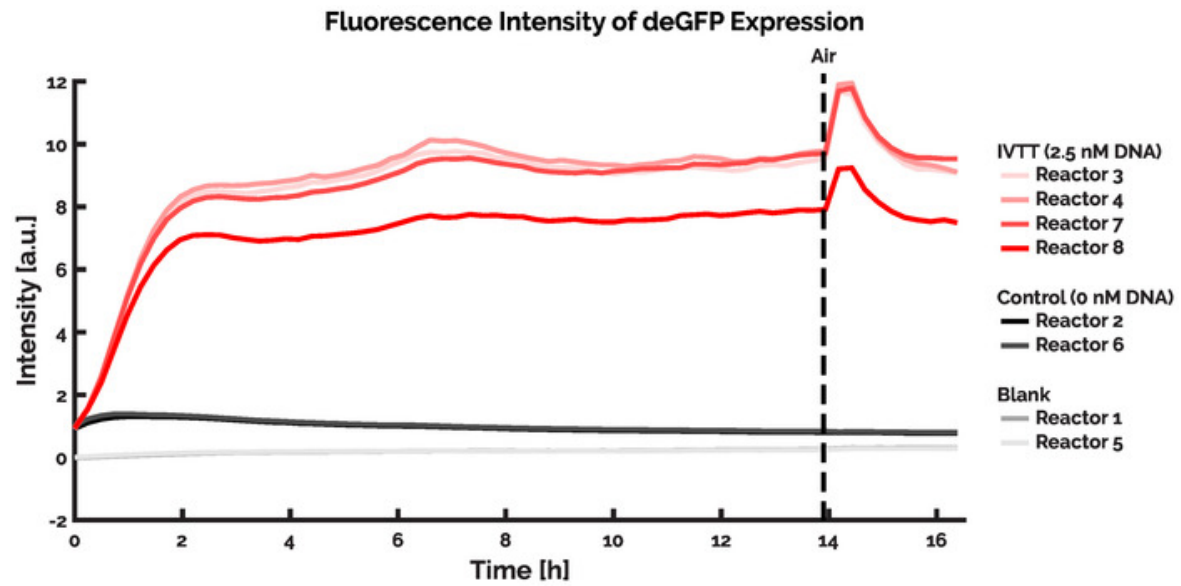

Figure 9. Results of an IVTT experiment expressing the deGFP protein. A prolonged IVTT reaction was initiated such that $30 \%$ of the reactor volume is displaced every 14.6 minutes. The reaction was allowed to run for over 16 hours before being terminated. Two reactors of the microfluidic device were used as blanks, with only ultrapure water being flown through the reactors throughout the experiment (reactors 1 and 5 ). All the other reactors comprised $75 \%$ IVTT reaction solution and $25 \%$ of either ultrapure water (reactors 2 and 6 ) or $2.5 \mathrm{nM}$ linear DNA templates coding for the expression of deGFP (reactors $3,4,7$, and 8 ). In all four reactors where DNA was added, there is clear deGFP expression. Three of the four reactors provide similar fluorescence intensity, with one reactor displaying lower fluorescence signal. This could be caused by a disparity in flow resulting in less DNA entering the reactor, or due to variations in the reactor dimensions. After 14 hours, a sudden increase is seen in the signal of the reactors containing DNA. This is caused by an air bubble entering the flow layer of the microfluidic device, presumably originating from one of the inflow solutions. The trapping of air in the microfluidic device significantly limits the flow of fluids through the channels, whereby no fresh reagents can be added to or removed from the reactors until the air has passed. Upon resumption of flow, the experiment returns to its previous fluorescence intensity. Please click here to view a larger version of this figure.

\section{Discussion}

A PDMS-based multilayer microfluidic device has been presented, and its capability to sustain IVTT reactions for prolonged periods of time has been demonstrated. Although well-suited for this specific example, this technology can conceivably be used for numerous other applications. The additional control over fluid flow - paired with the ability to continuously replenish reaction reagents whilst removing (by)products - is ideal for continuous synthesis reactions, the investigation of various dynamic behaviors, and the simultaneous conduction of multiple variations of a single reaction.

Despite the relatively straightforward fabrication process of PDMS based devices, the use thereof requires an extensive hardware setup. Comprising valve arrays, pressure regulators, pressure pumps, incubators, and cooling units, the transition from fabrication to use is not elementary, and requires a significant initial investment. In addition, the ability to consistently set-up and perform successful experiments with these devices requires a significant time-investment; a point which this manuscript aims to address. However, once in place, the entire setup can be modified for a range of purposes. Furthermore, the hardware setup comprises numerous modular elements, each of which can be expanded 
to allow more complex microfluidic device designs to be employed. Additionally, the modular design enables the replacement of hardware components by similarly functioning alternatives, such that users are not limited to the specific setup described here ${ }^{48,49}$.

Variability between individual devices, and in the external conditions (such as pressure fluctuations) can result in inaccuracies when performing experiments using these devices. To address this issue, a calibration of the system should be performed prior to each experiment, providing a unique Refresh Ratio for each of the reactors. Whilst the calibration addresses the device-to-device and experiment-to-experiment variations, it is a time consuming process and not flawless. Fluids with differing viscosities will not flow with the same rate when exposed to identical pressure, and as such performing the calibration with multiple reagents may not yield identical Refresh Ratios. This effect is attenuated by utilising three control channels to peristaltically pump the reagents into the microfluidic device, as opposed to regulating the flow by varying the supplied pressure only. As a last resort in cases where the disparity in viscosity is very large, a unique Refresh Ratio can be implemented for each individual reagent by performing multiple calibration experiments.

The use of a peristaltic pump to inject reagents into the microfluidic device attenuates the effects of using solutions with varying viscosities, however it also creates a secondary problem. Using discrete steps to pump fluids into the microfluidic device, means that the resolution of injections into a single reactor, is limited by the volume injected when performing a single pump cycle. Within our research this value determined during the calibration - is approximately equal to $1 \%$, indicating that a single pump cycle displaces approximately $1 \%$ of the reactor volume (about $0.1 \mathrm{~nL}$ ). As such, displacing $30 \%$ of the reactor volume requires the execution of 30 pump cycles, with 23 pump cycles of the IVTT reaction solution being added, and only 7 pump cycles of DNA or ultrapure water being added. Although sufficient for our research, alternative experimental protocols may encounter problems when attempting to add larger numbers of unique reagents, use a lower Refresh Fraction, or add smaller volumes of a single reagent to a reactor. In such cases, the microfluidic device design can be adapted to provide reactors with a larger volume. An example of such is reported in Niederholtmeyer et al. ${ }^{36}$.

Crucially, the device outlined within this manuscript allows reactions to be sustained for prolonged durations resulting in steady-state transcription and translation rates. By periodically injecting new reagents into the reactors - and removing reaction (by)products - the reactions are sustained and complex dynamic behaviors can be monitored. In this way, a platform has been created that - to some extent - mimics the cellular environment. Furthermore, this platform enables the exploration of the system dynamics, by adapting the period between injections and the specific composition of the injections. As a result, these multilayer microfluidic devices are a powerful tool for the characterisation and optimisation of novel synthetic networks which display complex dynamic behavior.

\section{Disclosures}

The authors declare that they have no competing financial interests.

\section{Acknowledgments}

This work was supported by the European Research Council, ERC (project n. 677313 BioCircuit) an NWO-VIDI grant from the Netherlands Organization for Scientific Research (NWO, 723.016.003), funding from the Ministry of Education, Culture and Science (Gravity programs, 024.001.035 \& 024.003.013), the Human Frontier Science Program Grant RGP0032/2015, the European Research Council under the European Union's Horizon 2020 research and innovation program Grant 723106, and a Swiss National Science Foundation Grant 200021 182019.

\section{References}

1. van Roekel, H. W. H. et al. Programmable chemical reaction networks: emulating regulatory functions in living cells using a bottom-up approach. Chemical Society Reviews. 44, 7465-7483 (2015).

2. Hori, Y., Kantak, C., Murray, R.M., Abate, A.R. Cell-free extract based optimization of biomolecular circuits with droplet microfluidics. Lab on a Chip. (2017).

3. Del Vecchio, D., Sontag, E. D. Dynamics and control of synthetic bio-molecular networks. in Proceedings of the American Control Conference. 1577-1588 (2007).

4. Purnick, P. E. M., Weiss, R. The second wave of synthetic biology: from modules to systems. Nature Reviews Molecular Cell Biology. 10, 410-422 (2009).

5. Padirac, A., Fujii, T., Rondelez, Y. Bottom-up construction of in vitro switchable memories. Proceedings of the National Academy of Sciences of the United States of America. 109, E3212-E3220 (2012).

6. Guido, N. J. et al. A bottom-up approach to gene regulation. Nature. 439, 856-860 (2006).

7. Genot, A. J., Fujii, T., Rondelez, Y. In vitro regulatory models for systems biology. Biotechnology Advances. 31, 789-796 (2013).

8. Elowitz, M. B., Leibler, S. A synthetic oscillatory network of transcriptional regulators. Nature. 403, 335-338 (2000).

9. Gardner, T. S., Cantor, C. R., Collins, J. J. Construction of a genetic toggle switch in Escherichia coli. Nature. 403, 339-342 (2000).

10. Montagne, K., Plasson, R., Sakai, Y., Fujii, T., Rondelez, Y. Programming an in vitro DNA oscillator using a molecular networking strategy. Molecular Systems Biology. 7, 466-466 (2014).

11. Khalil, A. S., Collins, J. J. Synthetic biology: applications come of age. Nature Reviews Genetics. 11, 367-379 (2010).

12. Hockenberry, A. J., Jewett, M. C. Synthetic in vitro circuits. Current Opinion in Chemical Biology. 16, 253-259 (2012).

13. Vecchio, D. Del. Modularity, context-dependence, and insulation in engineered biological circuits. Trends in Biotechnology. 33, 111-119 (2015).

14. Cardinale, S., Arkin, A. P. Contextualizing context for synthetic biology - identifying causes of failure of synthetic biological systems. Biotechnology Journal. 7, 856-866 (2012).

15. Venturelli, O. S. et al. Programming mRNA decay to modulate synthetic circuit resource allocation. Nature Communications. 8, 1-11 (2017).

16. Scott, M., Mateescu, E. M., Zhang, Z., Hwa, T. Interdependence of cell growth and gene expression: origins and consequences. Science. 330, 1099-1103 (2010). 
17. Borkowski, O., Ceroni, F., Stan, G. B., Ellis, T. Overloaded and stressed: whole-cell considerations for bacterial synthetic biology. Current Opinion in Microbiology. 33, 123-130 (2016).

18. Liao, C., Blanchard, A. E., Lu, T. An integrative circuit-host modelling framework for predicting synthetic gene network behaviours. Nature Microbiology. (2017).

19. Noireaux, V., Bar-Ziv, R., Libchaber, A. Principles of cell-free genetic circuit assembly. Proceedings of the National Academy of Sciences of the United States of America. 100, 12672-12677 (2003).

20. Shimizu, Y. et al. Cell-free translation reconstituted with purified components. Nature Biotechnology. 19, 751-755 (2001).

21. Oberholzer, T., Nierhaus, K. H., Luisi, P. L. Protein expression in liposomes. Biochemical and Biophysical Research Communications. 261, 238-241 (1999).

22. Yelleswarapu, M. et al. Sigma factor-mediated tuning of bacterial cell-free synthetic genetic oscillators. ACS Synthetic Biology. 7 , acssynbio.8b00300 (2018).

23. Karig, D. K., Iyer, S., Simpson, M. L., Doktycz, M. J. Expression optimization and synthetic gene networks in cell-free systems. Nucleic Acids Research. 40, 3763-3774 (2012).

24. Dunlop, M. J. et al. Distinct timescales of RNA regulators enable the construction of a genetic pulse generator. Biotechnology and Bioengineering. (2019).

25. Yoshiyama, T., Ichii, T., Yomo, T., Ichihashi, N. Automated in vitro evolution of a translation-coupled RNA replication system in a droplet flow reactor. Scientific Reports. 8, 1-8 (2018).

26. Iyer, S., Karig, D. K., Norred, S. E., Simpson, M. L., Doktycz, M. J. Multi-input regulation and logic with T7 promoters in cells and cell-free systems. PLoS ONE. 8, 1-12 (2013).

27. de los Santos, E. L. C., Meyerowitz, J. T., Mayo, S. L., Murray, R. M. Engineering Transcriptional Regulator Effector Specificity Using Computational Design and In vitro Rapid Prototyping: Developing a Vanillin Sensor. ACS Synthetic Biology. 5, 287-295 (2016).

28. Guo, S., Murray, R. M. Construct functional feedforward loop biological circuits in a cell-free system and in cells. ACS Synthetic Biology. acssynbio.8b00493 (2019).

29. Hughes, R. A., Ellington, A. D. Synthetic DNA synthesis and assembly: putting the synthetic in synthetic biology. Cold Spring Harbor Perspectives in Biology. 9, (2017).

30. Garamella, J., Marshall, R., Rustad, M., Noireaux, V. The all E. coli TX-TL toolbox 2.0: A platform for cell-free synthetic biology. ACS Synthetic Biology. 5, 344-355 (2016).

31. Takahashi, M. K. et al. Characterizing and prototyping genetic networks with cell-free transcription-translation reactions. Methods. 86, $60-72$ (2015).

32. Lee, J. W. et al. Creating single-copy genetic circuits. Molecular Cell. 63, 329-336 (2016).

33. Gibson, D. G. et al. Complete chemical synthesis, assembly, and cloning of a mycoplasma genitalium genome. Science. 319, 1215-1220 (2008).

34. Sun, Z. Z., Yeung, E., Hayes, C. A., Noireaux, V., Murray, R. M. Linear DNA for rapid prototyping of synthetic biological circuits in an Escherichia coli based TX-TL cell-free system. ACS Synthetic Biology. 3, 387-397 (2014).

35. Georgi, V. et al. On-chip automation of cell-free protein synthesis: new opportunities due to a novel reaction mode. Lab on a Chip. 16, 269-281 (2016).

36. Niederholtmeyer, H., Stepanova, V., Maerkl, S. J. Implementation of cell-free biological networks at steady state. Proceedings of the National Academy of Sciences of the United States of America. 110, 15985-15990 (2013).

37. A. S. Spirin, V. I. Baranov, L. A. Ryabova, S. Y. Ovodov, and Y. B. A. A continuous cell-free translation system capable of producing polypeptides in high yield. Science. 242, 1162-1164 (1988).

38. Karzbrun, E., Tayar, A. M., Noireaux, V., Bar-Ziv, R. H. Programmable on-chip DNA compartments as artificial cells. Science. 345, 829-832 (2014).

39. Timm, A. C., Shankles, P. G., Foster, C. M., Doktycz, M. J., Retterer, S. T. Toward microfluidic reactors for cell-free protein synthesis at the point-of-care. Small. 12, 810-817 (2016).

40. Doktycz, M. J., Foster, C. M., Retterer, S. T., Timm, A. C., Shankles, P. G. Characterization of extended channel bioreactors for continuousflow protein production. Journal of Vacuum Science \& Technology B, Nanotechnology and Microelectronics: Materials, Processing, Measurement, and Phenomena. 33, 06FM02 (2015).

41. Khnouf, R., Beebe, D. J., Fan, Z. H. Cell-free protein expression in a microchannel array with passive pumping. Lab on a chip. 9, 56-61 (2009).

42. Siuti, P., Retterer, S. T., Doktycz, M. J. Continuous protein production in nanoporous, picolitre volume containers. Lab on a Chip. 11, 3523-3529 (2011).

43. Kaminski, T. S., Scheler, O., Garstecki, P. Droplet microfluidics for microbiology: techniques, applications and challenges. Lab on a Chip. 16, 2168-2187 (2016).

44. Shin, J., Noireaux, V. An E. coli cell-free expression toolbox: Application to synthetic gene circuits and artificial cells. ACS Synthetic Biology. 1, 29-41 (2012).

45. Niederholtmeyer, $\mathrm{H}$. et al. Rapid cell-free forward engineering of novel genetic ring oscillators. eLife. 4, 1-18 (2015).

46. Galas, J., Haghiri-Gosnet, A.-M., Estévez-Torres, A. A nanoliter-scale open chemical reactor. Lab on a Chip. 13, 415-423 (2013).

47. Balaban, N. Q., Merrin, J., Chait, R., Kowalik, L., Leibler, S. Monolithic Microfabricated Valves and Pumps by Multilayer Soft Lithography. Science. 288, 113-116 (2004).

48. Brower, K. et al. An open-source, programmable pneumatic setup for operation and automated control of single- and multi-layer microfluidic devices. HardwareX. 3, 117-134 (2018).

49. White, J. A., Streets, A. M. Controller for microfluidic large-scale integration. HardwareX. 3, 135-145 (2018). 